\title{
ORIGINAL
}

\section{EVOLUCIÓN DE LOS EFECTOS DE LAS TEMPERATURAS MÁXIMAS SOBRE LA MORTALIDAD POR CAUSAS ORGÁNICAS EN CASTILLA- LA MANCHA DE 1975 A 2003(*)}

\author{
Isidro J. Mirón Pérez (1), Juan Carlos Montero Rubio (1), Juan José Criado- Álvarez (1), Sheila \\ Mayoral Arenas (2) Julio Díaz Jiménez (3) y Cristina Linares Gil (3).
}

(1) Consejería de Sanidad, Junta de Comunidades de Castilla- La Mancha.

(2) Sociedad Castellanomanchega de Medicina Preventiva y Salud Pública.

(3) Fundación General de la Universidad Autónoma de Madrid para el Dpto. de Educación para el Desarrollo Sostenible del Ayuntamiento de Madrid.

\section{RESUMEN}

Fundamento: La relación no lineal temperatura- mortalidad varía según las características de la zona geográfica estudiada. En determinados lugares un incremento en el nivel de desarrollo ha conducido a una menor influencia de las variables ambientales sobre la mortalidad. Se analiza la evolución entre 1975 y 2003 de la asociación de las temperaturas máximas con la mortalidad por causas orgánicas en Castilla-La Mancha.

Métodos: Los datos diarios de temperaturas máximas y de mortalidad por causas orgánicas se dividen en tres periodos: 1975-1984, 1985-1994 y 1995-2003. Tras un preblanqueo de los datos aplicando el modelo ARIMA ajustado para las series de temperaturas, se calculan las funciones de correlación cruzada entre los residuos de las series de temperaturas y de mortalidad con 7 desfases en verano y 15 en invierno, comparándose los coeficientes de correlación.

Resultados: Se observa en los meses calurosos un incremento del número de retardos significativos $(\mathrm{p}<0,05)$ de la primera a la segunda década de estudio en el conjunto regional, con algunas diferencias provinciales. En la tercera década el número de lags significativos varía ligeramente, incrementándose los coeficientes de correlación cruzada de forma significativa $(p<0,05)$ para el desfase 3 en Toledo y en el total regional.

Conclusiones: La asociación de las temperaturas máximas con la mortalidad por causas orgánicas se ha ampliado e intensificado desde la década 1975-1984. El envejecimiento de la población podría haber contrarrestado el posible efecto beneficioso del crecimiento económico sobre esta relación. En los meses fríos no se encuentra evolución temporal apreciable.

Palabras clave: Temperaturas máximas. Mortalidad. Evolución temporal. Envejecimiento.

\section{Correspondencia:}

Juan Carlos Montero Rubio

Sección de Microbiología Clínica y Ambiental

Servicio de Laboratorio

Instituto de Ciencias de la Salud de Castilla- La Mancha

Ctra. de Extremadura km 114.

45600 Talavera de la Reina (Toledo)

Correo electrónico: jcmontero@jccm.es

\section{ABSTRACT \\ Trends in Effects of Maximum Temperatures on Organic-Cause Mortality in Castile- La Mancha, Spain from 1975 to 2003}

Background: Non-lineal temperature-mortality relationship varies depending on the characteristics of the designated study geographic zone. In given places, a growing level of economic development has led to lesser influence of environmental variables on mortality. This paper analyzes trends in the association between maximum temperatures and organic-cause mortality from 1975 to 2003 in Castile- La Mancha (Spain).

Methods: Daily maximum temperatures and organic-cause mortality data were divided into 3 time period: $1975-1984,1985-$ 1994 and 1995-2003. After data pre-whitening by applying ARIMA model estimated for the daily maximum temperature series, we calculate cross-correlation functions between residuals of temperature and mortality, 7 days lagged for summer, 15 for cold months, and comparing its correlation coefficients.

Results: We observe an increasing number of significant lags during the warm season $(\mathrm{p}<0.05)$ between first and second decades studied in regional overall but with some provincial differences. In the third study period the number of significant lags varies slightly, although cross correlation coefficients were significantly upward $(p<0.05)$ at lag 3 in the entire region and Toledo in particular.

Conclusions: Maximum temperature and mortality by organic cause association became more extensive and intense since 1975-1984 decade in Castile- La Mancha. The aging of regional population could offset the probable beneficial effect of economic growth on this relationship. No appreciable time trends are found in cold months.

Key words: Maximum temperatures. Mortality. Time trends. Aging.

(*) Este trabajo se ha financiado con una ayuda a la Sociedad Castellanomanchega de Medicina Preventiva y Salud Pública de la Consejería de Sanidad de la Junta de Comunidades de Castilla-La Mancha (Expediente PI 22/2003). 


\section{INTRODUCCIÓN}

La influencia de la temperatura del aire sobre la mortalidad está ampliamente descrita como una relación no lineal, en forma de "V", debido a que la mortalidad aumenta a medida que sube o baja la temperatura ambiental a partir de una determinada temperatura de confort o de mínima mortalidad ${ }^{1-5}$. Según las características climáticas de la zona geográfica estudiada la intensidad de esta relación puede variar como consecuencia de un proceso de adaptación de la población a su entorno ${ }^{6,7}$. De hecho, se ha observado que en las ciudades con temperaturas medias anuales más bajas se produce una mayor mortalidad por calor $y$, por el contrario, cuanto mayor es la influencia de la época estival mayor es la mortalidad por frío ${ }^{8}$. Así mismo, los efectos del calor pueden ser más inmediatos o más retrasados según la situación geográfica y, quizás, socio- sanitaria de la ciudad estudiada9 ${ }^{9}$. Incluso cambios lo suficientemente rápidos en el entorno podrían modificar de alguna manera la relación de la temperatura y otros factores ambientales con la mortalidad. En este sentido, se ha comprobado que en la medida en que se alcanzaba un mayor desarrollo económico se iba suavizando la influencia de las variables ambientales sobre la morbi-mortalidad $^{10}$. En estudios más recientes se ha descrito cómo en algunas zonas la influencia de las altas temperaturas sobre la mortalidad se ha modificado a lo largo del tiempo, tendiendo hacia una paulatina menor asociación calor- mortalidad ${ }^{11,12}$.

La confirmación de que nos dirigimos hacia un significativo incremento de temperaturas a nivel global, tal y como advierte en sus últimas reuniones el Panel Intergubernamental para el Cambio Climático (IPCC), junto con las previsiones locales realizadas para España sobre un aumento de las temperaturas medias y de una mayor frecuencia de días con temperaturas extre- mas, sobre todo en verano ${ }^{13}$, aconseja la realización de estudios que permitan anticiparse a potenciales consecuencias de este proceso sobre la salud pública. Debido a estas estimaciones, diversos expertos vienen pronosticando desde hace tiempo un incremento de la mortalidad inducida por el calentamiento global ${ }^{14,15}$, aunque partiendo de modelos que no contemplan las posibles variaciones en el entorno físico y social de la población.

Por otra parte, casi todos los trabajos realizados hasta ahora se sitúan en ciudades o áreas geográficas reducidas, donde un alto número de habitantes se encuentra expuesto a condiciones climáticas similares, por lo que sería interesante aplicar una metodología que permita abordar este tipo de análisis en áreas geográficas más extensas, con densidades de población bajas, como es el caso de Castilla-La Mancha (CLM), en las que de otra forma sería difícil alcanzar resultados con significación estadística suficiente.

Son escasos los estudios realizados hasta ahora en España que constaten una evolución de la asociación temperatura- mortalidad a lo largo del tiempo, siendo aventurado deducir tendencias similares por el hecho de presentar también niveles de desarrollo económico positivos en los últimos años, ya que podrían darse circunstancias locales específicas que influyeran en esta relación.

Puesto que la información de que se dispone en el ámbito de CLM acerca de la influencia de las temperaturas sobre indicadores sanitarios es muy limitada, los datos que se aportan pretenden ser novedosos y útiles para una adecuada planificación sanitaria regional y para posteriores estudios que profundicen en el conocimiento de esta relación.

Por tanto, el objetivo de este estudio es determinar la evolución entre 1975 y 2003 
de la asociación de las temperaturas máximas con la mortalidad por causas orgánicas en CLM.

\section{MATERIAL Y MÉTODOS}

Los datos de mortalidad diaria proceden del Registro de Mortalidad de CLM, dependiente de la Consejería de Sanidad y que recoge todas las muertes ocurridas en CLM entre los años 1975 y 2003, que son los registros disponibles. Estos datos están codificados según la Clasificación Internacional de Enfermedades (CIE 8: 1975-1979, CIE 9: 1980-1998 y CIE 10: 1999-2003). Se trata de datos provinciales de mortalidad diaria por causas orgánicas (todas excepto externas). Para la CIE-8 y CIE-9 se han usado los códigos 001-799 y para la CIE-10, A00-R99. Se excluyen las causas externas por presentar éstas una débil relación con la temperatura.

Los registros diarios de temperaturas del periodo 1975-2003 fueron suministrados por el Instituto Nacional de Meteorología (INM). Se eligen los correspondientes a los observatorios situados en las capitales de provincia (Tabla 1) a partir de 42 estaciones meteorológicas seleccionadas en base a un estudio previo en el que se sometieron los datos de temperaturas máximas, medias y mínimas diarias a un proceso sucesivo de relleno de lagunas, detección de discontinuidades, homogenización y análisis factorial por componentes principales, resultando toda la región de CLM un único clúster en función de las temperaturas, lo que también permite la desagregación de datos a nivel provincial y la utilización de las estaciones de las capitales como referencia ${ }^{16}$.

En el presente estudio se opta por utilizar las series de temperaturas máximas diarias porque se pretende seguir una línea de investigación relacionada principalmente con los efectos del calor sobre la mortalidad de la población en esta región. Así mismo, los resultados obtenidos podrían compararse con los de regiones vecinas, como Madrid, donde en estudios realizados sobre olas de calor se utilizaron series de temperaturas máximas diarias. Además, probablemente sea mucho más comprensible para la población expresar, en su caso, medidas preventivas relacionadas con el calor en términos de temperaturas máximas que de temperaturas medias o mínimas.

Para estudiar la evolución temporal de la asociación temperaturas máximasmortalidad se establecen tres periodos de

Tabla 1

Estaciones meteorológicas seleccionadas. Guadalajara "Serranillo" y Toledo "Buenavista" son continuación de Guadalajara "Instituto" y Toledo "Lorenzana", respectivamente, por cambio de ubicación de las mismas denominándose la unión de los registros pre y post deslocalización Guadalajara "Compuesta" y Toledo "Compuesta"16

\begin{tabular}{|l|l|c|c|c|l|}
\hline Código INM & \multicolumn{1}{|c|}{ Nombre Estación } & Longitud & Latitud & Altitud (m) & \multicolumn{1}{|c|}{ Provincia } \\
\hline 8175 & Albacete "Los Llanos Base Aérea" & $01-51-47 \mathrm{~W}$ & $38-57-08$ & 704 & Albacete \\
\hline 4121 & Ciudad Real Escuela de Magisterio & $03-55-11 \mathrm{~W}$ & $38-59-22$ & 627 & Ciudad Real \\
\hline 8096 & Cuenca & $02-08-17 \mathrm{~W}$ & $40-04-00$ & 956 & Cuenca \\
\hline $3168 \mathrm{C}$ & Guadalajara "Serranillo" & $03-10-27 \mathrm{~W}$ & $40-39-40$ & 635 & Guadalajara \\
\hline $3168 \mathrm{~A}$ & Guadalajara "Instituto" & $03-09-52 \mathrm{~W}$ & $40-38-05$ & 685 & Guadalajara \\
\hline 3259 & Toledo "Lorenzana" & $04-01-25 \mathrm{~W}$ & $39-51-40$ & 540 & Toledo \\
\hline $3260 \mathrm{~B}$ & Toledo "Buenavista" & $04-02-58 \mathrm{~W}$ & $39-53-05$ & 516 & Toledo \\
\hline
\end{tabular}


Tabla 2

Parámetros descriptivos de la serie de temperaturas máximas diarias $\left({ }^{\circ} \mathrm{C}\right)$ (Toledo "Compuesta") y de la mortalidad diaria por causas orgánicas en Castilla-La Mancha

\begin{tabular}{|c|c|c|c|c|c|c|c|}
\hline & Max. & Mín. & Med. & D. Standard & Varianza & Tendencia & Periodicidad \\
\hline $\mathrm{T}^{a}$ máxima & 42,4 & $-0,4$ & 21,7 & 8,829 & 77,951 & NO & anual, semestral, días (3,7) \\
\hline Orgánicas & 85 & 16 & 39,81 & 8,579 & 73,593 & SI & anual, semestral, trimestral, días (3) \\
\hline
\end{tabular}

comparación: 1975-1984; 1985-1994 y 1995-2003 (tres décadas, la última de 9 años). De este modo, dispondremos de tres grupos de datos de temperaturas máximas diarias y de mortalidad diaria por causas orgánicas en el conjunto regional y por cada una de las 5 provincias de CLM.

Las relaciones estadísticas causales entre las temperaturas máximas diarias y la mortalidad diaria por causas orgánicas se han establecido previa aplicación del modelo ARIMA estimado para las series de temperaturas máximas diarias a éstas y a las series mortalidad diaria (preblanqueo), siguiendo el Método de Box-Jenkins ${ }^{17}$. Para facilitar este proceso se realiza una detección previa de periodicidades mediante el análisis espectral de las series. De esta manera, durante el procedimiento de modelización quedará controlado el comportamiento estacional análogo que puedan presentar las series (Tabla 2), así como la tendencia puesto que es corregida, en su caso, mediante la correspondiente diferenciación. Con los residuos obtenidos de ambas series se calculan las funciones de correlación cruzada (FCC) con 7 retardos o desfases para los meses cálidos (junio, julio, agosto y septiembre) y 15 para los fríos (noviembre, diciembre, enero, febrero y marzo) y para cada década establecida. De esta manera, se obtendrá en qué días después de producirse una temperatura cálida o una temperatura fría la asociación temperatura máxima- mortalidad es significativa $(\mathrm{p}<0,05)$, en términos cualitativos, así como la evolución de la misma lo largo del tiempo. Así mismo, comparando los intervalos de confianza $(\mathrm{p}<0,05)$ de los coeficientes de correlación generados por las FCC podrá apreciarse si esa relación se ha intensificado con el tiempo de forma significativa.

Se utiliza en el análisis el paquete estadístico SPSS.

\section{RESULTADOS}

Considerando la mortalidad por causas orgánicas de la Comunidad de CLM y tomando como estación termométrica de referencia la de Toledo "Compuesta" (unión de Toledo "Lorenzana" y Toledo "Buenavista"16), puede apreciarse en la Tabla 3 una creciente evolución de la asociación temperatura- mortalidad durante los meses de verano (de junio a septiembre), ya que de 4 desfases o lags significativos (1, 2, 3 y 4) en la década 1975-1984 se pasa a $5(0,1,2,3$ y 5$)$ en la década 1985-1994 y nuevamente 4 (1, 2, 3 y 4), aunque con mayores coeficientes de correlación, en el periodo 1995-2003. En éste, considerando sus FCC, el coeficiente de correlación del lag 3 ha aumentado significativamente respecto a las dos décadas anteriores.

La evolución decenal presenta algunas variaciones en el número de desfases significativos cuando se desagrega la mortalidad por causas orgánicas a nivel provincial, siendo los datos termométricos de referencia los suministrados por las estaciones de las respectivas capitales de pro- 
Tabla 3

Evolución de los coeficientes de correlación e intervalos de confianza de las FFC entre residuos de temperaturas máximas diarias y de mortalidad por causas orgánicas en los desfases con significación estadística $(\mathbf{p}<0,05)$ en alguno de los periodos considerados durante los meses cálidos (junio-septiembre)

\begin{tabular}{|c|c|c|c|c|}
\hline & Lag & $1975-1984$ & 1984-1994 & $1995-2003$ \\
\hline \multirow[t]{4}{*}{ Albacete } & 1 & $\begin{array}{c}\mathbf{0 , 1 0 1 *} \\
(0,044-0,158)\end{array}$ & $\begin{array}{c}\mathbf{0 , 0 8 1} * \\
(0,024-0,138)\end{array}$ & $\begin{array}{c}\mathbf{0 , 1 1 7 *} \\
(0,058-0,176)\end{array}$ \\
\hline & 2 & $\begin{array}{c}\mathbf{0 , 0 9 2} * \\
(0,035-0,150)\end{array}$ & $\begin{array}{c}0,041 \\
(-0,016-0,098)\end{array}$ & $\begin{array}{c}0,057 \\
(-0,002-0,116)\end{array}$ \\
\hline & 3 & $\begin{array}{c}\mathbf{0 , 0 9 4} * \\
(0,037-0,152)\end{array}$ & $\begin{array}{c}0,003 \\
(-0,054-0,060)\end{array}$ & $\begin{array}{c}0,038 \\
(-0,021-0,097)\end{array}$ \\
\hline & 4 & $\begin{array}{c}0,032 \\
(-0,025-0,089)\end{array}$ & $\begin{array}{c}0,041 \\
(-0,016-0,098)\end{array}$ & $\begin{array}{c}\mathbf{0 , 0 8 7} * \\
(0,028-0,146)\end{array}$ \\
\hline \multirow[t]{6}{*}{ Ciudad Real } & 0 & $\begin{array}{c}0,028 \\
(-0.029-0,085)\end{array}$ & $\begin{array}{c}0,054 \\
(-0,003-0,111)\end{array}$ & $\begin{array}{c}\mathbf{0 , 0 7 2} * \\
(0,013-0,131)\end{array}$ \\
\hline & 1 & $\begin{array}{c}\mathbf{0 , 0 7 2} * \\
(0,015-0,129)\end{array}$ & $\begin{array}{c}\mathbf{0 , 1 1 7} * \\
(0,060-0,174)\end{array}$ & $\begin{array}{c}\mathbf{0 , 0 9 4} * \\
(0,035-0,153)\end{array}$ \\
\hline & 2 & $\begin{array}{c}\mathbf{0 , 0 8 1} * \\
(0,024-0,138)\end{array}$ & $\begin{array}{c}\mathbf{0 , 1 6 0 *} \\
(0,103-0,217)\end{array}$ & $\begin{array}{c}\mathbf{0 , 1 5 2} * \\
(0,093-0,211)\end{array}$ \\
\hline & 3 & $\begin{array}{c}\mathbf{0 , 0 7 3} * \\
(0,016-0,130)\end{array}$ & $\begin{array}{c}\mathbf{0 , 1 1 1} * \\
(0,054-0,168)\end{array}$ & $\begin{array}{c}\mathbf{0 , 1 7 4} * \\
(0,115-0,233)\end{array}$ \\
\hline & 4 & $\begin{array}{c}\mathbf{0 , 0 6 7 *} \\
(0,010-0,124)\end{array}$ & $\begin{array}{c}\mathbf{0 , 0 8 1} * \\
(0,024-0,138)\end{array}$ & $\begin{array}{c}\mathbf{0 , 0 6 3 *} \\
(0,004-0,122)\end{array}$ \\
\hline & 5 & $\begin{array}{c}\mathbf{0 , 0 5 9} * \\
(0,002-0,116)\end{array}$ & $\begin{array}{c}0,040 \\
(-0,017-0,097)\end{array}$ & $\begin{array}{c}0,049 \\
(-0,009-0,108)\end{array}$ \\
\hline \multirow[t]{4}{*}{ Cuenca } & 1 & $\begin{array}{c}0,053 \\
(-0.004-0,110)\end{array}$ & $\begin{array}{c}\mathbf{0 , 0 9 0 *} \\
(0,033-0,147)\end{array}$ & $\begin{array}{c}0,030 \\
(-0,029-0,089)\end{array}$ \\
\hline & 2 & $\begin{array}{c}0,011 \\
(-0,046-0,068)\end{array}$ & $\begin{array}{c}\mathbf{0 , 0 9 7} * \\
(0,040-0,154)\end{array}$ & $\begin{array}{c}\mathbf{0 , 1 0 2} * \\
(0,043-0,161)\end{array}$ \\
\hline & 3 & $\begin{array}{c}0,023 \\
(-0,034-0,080)\end{array}$ & $\begin{array}{c}0,031 \\
(-0,034-0,080)\end{array}$ & $\begin{array}{c}\mathbf{0 , 0 9 9} \\
(0,040-0,158)\end{array}$ \\
\hline & 7 & $\begin{array}{c}0,003 \\
(-0.054-0,060) \\
\end{array}$ & $\begin{array}{c}0,024 \\
(-0,033-0,081) \\
\end{array}$ & $\begin{array}{c}\mathbf{0 , 0 8 2} * \\
(0,023-0,141) \\
\end{array}$ \\
\hline \multirow[t]{5}{*}{ Guadalajara } & 0 & $\begin{array}{c}-0,011 \\
(-0,068-0,046)\end{array}$ & $\begin{array}{c}\mathbf{0 , 0 6 2} * \\
(0,005-0,119)\end{array}$ & $\begin{array}{c}0,027 \\
(-0,032-0,086)\end{array}$ \\
\hline & 1 & $\begin{array}{c}\mathbf{0 , 0 6 9 *} \\
(0,012-0,126) \\
\end{array}$ & $\begin{array}{c}\mathbf{0 , 0 6 2} * \\
(0,005-0,119) \\
\end{array}$ & $\begin{array}{c}0,031 \\
(-0,028-0,090) \\
\end{array}$ \\
\hline & 2 & $\begin{array}{c}0,036 \\
(-0,021-0,093)\end{array}$ & $\begin{array}{c}0,053 \\
(-0.004-0,110)\end{array}$ & $\begin{array}{c}\mathbf{0 , 0 8 0 *} \\
(0,021-0,139)\end{array}$ \\
\hline & 3 & $\begin{array}{c}-0,002 \\
(-0,059-0,055)\end{array}$ & $\begin{array}{c}-0,007 \\
(-0,064-0,050)\end{array}$ & $\begin{array}{c}\mathbf{0 , 0 6 7 *} \\
(0,008-0,126)\end{array}$ \\
\hline & 6 & $\begin{array}{c}0,031 \\
(-0,034-0,080)\end{array}$ & $\begin{array}{c}0,002 \\
(-0,055-0,059)\end{array}$ & $\begin{array}{c}\mathbf{0 , 0 5 9} * \\
(0,0002-0,118)\end{array}$ \\
\hline \multirow[t]{5}{*}{ Toledo } & 1 & $\begin{array}{c}0,052 \\
(-0,005-0,109)\end{array}$ & $\begin{array}{c}0,054 \\
(-0,003-0,111)\end{array}$ & $\begin{array}{c}\mathbf{0 , 0 9 9} * \\
(0,040-0,158)\end{array}$ \\
\hline & 2 & $\begin{array}{c}\mathbf{0 . 0 7 4} * \\
(0,017-0,131) \\
\end{array}$ & $\begin{array}{c}\mathbf{0 , 0 7 8}^{*} \\
(0,021-0,135) \\
\end{array}$ & $\begin{array}{c}\mathbf{0 , 1 6 2} * \\
(0,103-0,221) \\
\end{array}$ \\
\hline & 3 & $\begin{array}{c}0,043 \\
(-0,014-0,100)\end{array}$ & $\begin{array}{c}\mathbf{0 , 0 9 0 *} \\
(0,033-0,147)\end{array}$ & $\begin{array}{c}0,167 * \\
(0,108-0,226)^{* *}\end{array}$ \\
\hline & 4 & $\begin{array}{c}0,042 \\
(-0,015-0,099\end{array}$ & $\begin{array}{c}\mathbf{0 , 0 7 7} * \\
(0.020-0,134)\end{array}$ & $\begin{array}{c}\mathbf{0 , 0 8 7} * \\
(0,028-0,146)\end{array}$ \\
\hline & 7 & $\begin{array}{c}0,054 \\
(-0,003-0,111)\end{array}$ & $\begin{array}{c}0,004 \\
(-0.053-0,061)\end{array}$ & $\begin{array}{c}\mathbf{0 , 0 8 2} \\
(0,026-0,138)\end{array}$ \\
\hline \multirow[t]{6}{*}{ Castilla-La Mancha } & 0 & $\begin{array}{c}0,035 \\
(-0,022-0,092)\end{array}$ & $\begin{array}{c}\mathbf{0 , 0 6 0}^{*} \\
(0,003-0,117)\end{array}$ & $\begin{array}{c}0,032 \\
(-0,027-0,091)\end{array}$ \\
\hline & 1 & $\begin{array}{c}\mathbf{0 , 1 0 0 *} \\
(0,043-0,157)\end{array}$ & $\begin{array}{c}\mathbf{0 , 1 4 4} * \\
(0,087-0,201)\end{array}$ & $\begin{array}{c}\mathbf{0 , 1 8 5 *} \\
(0,126-0,244)\end{array}$ \\
\hline & 2 & $\begin{array}{c}\mathbf{0 , 1 4 7 *} \\
(0,090-0,204)\end{array}$ & $\begin{array}{c}\mathbf{0 , 1 7 7} * \\
(0,120-0,234)\end{array}$ & $\begin{array}{c}\mathbf{0 , 2 3 4} * \\
(0,175-0,293)\end{array}$ \\
\hline & 3 & $\begin{array}{c}\mathbf{0 , 0 7 3} * \\
(0,016-0,130) \\
\end{array}$ & $\begin{array}{c}\mathbf{0 , 0 9 6}^{*} \\
(0,039-0,153) \\
\end{array}$ & $\begin{array}{c}0,223 * \\
(0,164-0,282)^{* *}\end{array}$ \\
\hline & 4 & $\begin{array}{c}\mathbf{0 , 0 6 4} * \\
(0,007-0,121)\end{array}$ & $\begin{array}{c}0,048 \\
(-0,009-0,105)\end{array}$ & $\begin{array}{c}\mathbf{0 , 0 8 1 *} \\
(0,025-0,137)\end{array}$ \\
\hline & 5 & $\begin{array}{c}0,010 \\
(-0,047-0,067)\end{array}$ & $\begin{array}{c}\mathbf{0 , 0 7 4} * \\
(0,017-0,131)\end{array}$ & $\begin{array}{c}-0,003 \\
(-0,062-0,056)\end{array}$ \\
\hline
\end{tabular}

* Coeficiente de correlación significativo.

** Coeficiente de correlación significativamente superior al de alguna de las décadas anteriores. 
vincia. En general, el mayor incremento en el número de retardos significativos durante el verano se da entre el periodo 1975-1984 y 1985-1994, si bien en las provincias de Cuenca, Guadalajara y, sobre todo, Toledo se produce también un claro aumento en el tercer grupo de años, como se puede observar en dicha tabla y, de manera más clara en la Figura 1. Se aprecia en esta provincia que de 1 lag significativo en la primera década (2) se pasa a 3 en la segunda $(2,3$ y 4$)$ y 5 en el último periodo considerado (1, 2, 3, 4 y 7). Además, el coeficiente de correlación en el lag 3 es significativamente mayor en el tercer periodo respecto al primero (Tabla 3 ).

En la provincia de Albacete la evolución de la asociación temperaturas máximas- mortalidad en verano muestra un comportamiento diferente durante el periodo de tiempo considerado, ya que número de desfases significativos disminuye de 3 a 1 entre las dos primeras décaCiudad Real se pasa de 5 a 4 lags significativos, los coeficientes de correlación, al contrario que en Albacete, aumentan aunque no lo hagan de una forma estadísticamente significativa.

Durante los meses fríos (noviembremarzo) no se produce una evolución clara en los efectos de las temperaturas frías sobre la mortalidad por causas orgánicas, tanto considerando toda la región de CLM como a nivel provincial. Así, observamos que en la FCC para el conjunto de la Región (Figura 2) se mantiene el número de lags significativos (5) entre los tres periodos considerados, pero con la particularidad de que el desfase 15, siempre con coeficientes de correlación positivos, aparece estadísticamente significativo en la década 1985-1994, frente al signo negativo del resto de retrasos significativos. das, subiendo a 2 en la tercera. Aunque en

Figura 1

Funciones de correlaciones cruzadas entre residuos de mortalidad diaria por causas orgánicas en la provincia de Toledo y residuos de temperaturas máximas diarias de verano (junio-septiembre) en los periodos 1975-1984 (1a), 1985-1994 (1b) y 1995-2003 (1c). Estación termométrica de referencia: Toledo "Compuesta"
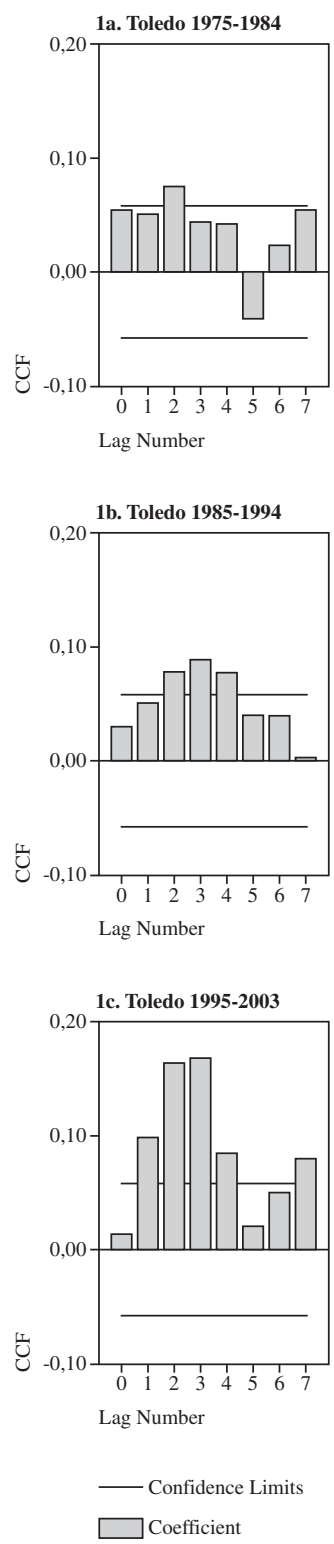
Figura 2

Funciones de correlaciones cruzadas entre residuos de mortalidad diaria por causas orgánicas en Castilla-La Mancha y residuos de temperaturas máximas diarias de los meses fríos (noviembre-marzo) en los periodos 1975-1984 (2a), 1985-1994 (2b) y 1995-2003 (2c). Estación termométrica de referencia: Toledo "Compuesta"
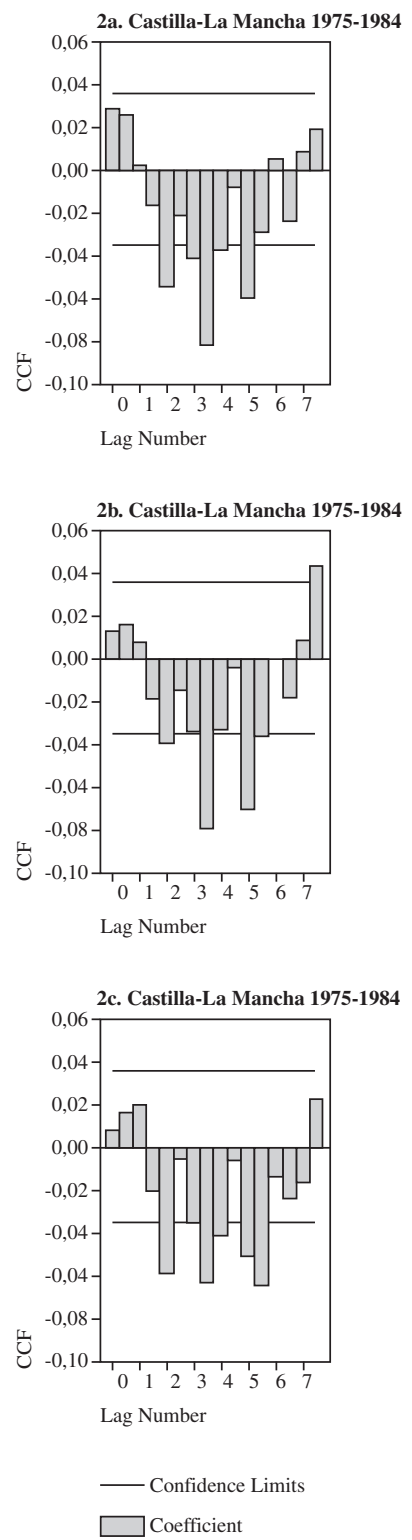

Rev Esp Salud Pública 2007, Vol. 81, N. ${ }^{\circ} 4$

\section{DISCUSIÓN}

El incremento del número de desfases o lags estadísticamente significativos indica que los efectos del calor sobre la mortalidad por causas orgánicas se han ampliado en CLM entre las décadas 1975-1984 y 19851994. Es decir, después de un evento caluroso se producen fallecimientos durante más días. En el periodo 1995-2003 la asociación evoluciona, en general, hacia un mantenimiento de la situación respecto a la anterior década (1985-1994), si bien con ligeros descensos o aumentos del número de retardos significativos según las provincias consideradas. El aumento de los coeficientes de correlación (excepto en Albacete) a lo largo del tiempo, significativos para el desfase 3 en el cómputo regional y en la provincia de Toledo, apunta hacia una intensificación de la asociación temperatura- mortalidad por causas orgánicas en CLM. Esto resulta coherente con lo obtenido en un reciente estudio sobre la evolución de la temperatura máxima de mínima mortalidad (temperatura de confort) en CLM ${ }^{18}$, en el que se describe cómo, excepto en Albacete, ésta ha disminuido de 1975 a 2003 por aumentar las tasas de mortalidad cuando la temperatura es cálida en mayor medida que cuando es fría.

Sin embargo, estos resultados entran en aparente contradicción respecto a estudios realizados en otros lugares, como Estados Unidos $^{19}$ y Londres $^{20}$, donde se describe una evolución decreciente de la mortalidad en relación con la temperatura, así como con otra serie de trabajos que relacionan mejoras de indicadores socio-económicos con una disminución de la influencia del calor sobre la mortalidad ${ }^{21-23}$, en la línea de lo que se había descrito antes en Japón como la "desperiodificación" de las enfermedades en sociedades avanzadas ${ }^{24}$, pudiendo interpretarse que la desaparición de la mortalidad estacional sería un indicador de alto nivel socio- económico en el área de estudio. 
Evidentemente esto no ha sido así en CLM, donde una favorable evolución de los indicadores económicos durante el periodo de estudio, en el que se incluye el proceso de integración de España en la CE, ha ido acompañada de un incremento de la asociación calor- mortalidad. Hay que señalar que el PIB regional por habitante del último año de cada periodo de estudio ha crecido de $3.223 €$ en 1984 a $8.275 €$ en 1994 y $13.911 €$ en 2003, según datos económicos y poblacionales del Instituto Nacional de Estadística (INE). Por tanto, deben existir factores específicos regionales que expliquen esta particular evolución.

Está descrito que en diferentes lugares la influencia de las temperatura del aire sobre la mortalidad, sobre todo por causas respiratorias y circulatorias, es más acusada en las personas de mayor edad ${ }^{25-27}$, incluyendo zonas limítrofes a CLM como Madrid ${ }^{28,29}$. En este sentido, hay que señalar que la evolución del porcentaje de población mayor de 65 años en CLM durante los 29 años que abarca el estudio ha sido creciente, pasando del 12,39\% al 19,75\%. La evolución ha sido similar en todas las provincias, por lo que factores específicos deben haber influido en la provincia de Albacete, ya que pese a producirse también un fuerte envejecimiento poblacional la evolución de la asociación temperatura- mortalidad ha sido diferente.

Así pues, es muy probable que sea este drástico aumento de la población mayor de 65 años la causa principal del efecto creciente de las temperaturas cálidas sobre la mortalidad por causas orgánicas en CLM, sin que el paralelo crecimiento económico y mejora de todo tipo de infraestructuras (sanitarias, aire acondicionado, etc.) de la Región haya sido suficiente para compensar el efecto negativo del envejecimiento de la población. Probablemente, si no hubiera coincidido esa etapa de alto crecimiento económico las consecuencias en términos de mortalidad podrían haber sido mayores.
Aun cuando los resultados muestran en las tres décadas un efecto rápido de las altas temperaturas sobre la mortalidad, concentrándose dentro de la primera semana tras el evento térmico (principalmente en los 4 primeros días), el aumento del número de días en los que se registra mortalidad significativa después de producirse una alta temperatura ambiental, puede indicar cierta menor inmediatez en las defunciones relacionadas con el calor causadas por el agravamiento de patologías subyacentes, quizás debido precisamente a la mejora de infraestructuras sanitarias a lo largo del periodo de estudio. El efecto de las temperaturas frías es más diferido, con desfases negativos significativos desde el $4^{\circ}$ hasta el $11^{\circ}$. Todo ello coincide con lo encontrado en estudios realizados en la vecina Comunidad de Madrid $^{30,31}$, donde también se observa el efecto rápido del calor y más retrasado del frío sobre la mortalidad, al igual que en otros lugares de Europa ${ }^{32,33}$ y Estados Uni$\operatorname{dos}^{34}$.

Por tanto, no se puede afirmar que exista una evolución interdecenal clara en el sentido de que los efectos del frío o del calor sobre la mortalidad por causas orgánicas se hayan adelantado o retrasado sino que los del calor se han extendido, sobre todo entre las dos primeras décadas. El efecto siega que podría estar indicando el lag 15 positivo cuando las temperaturas son bajas (significativo en la década 1985-1994 sin desagregación provincial) está descrito con mayor frecuencia durante la época caluro$\mathrm{sa}^{28,33,34}$, aunque no es inédito en los meses fríos $^{34}$.

Por otra parte, analizando las series de temperaturas máximas diarias podemos señalar cómo éstas han evolucionado hacia la ocurrencia de un mayor número de días calurosos. De hecho, si aplicamos la metodología aceptada por el Ministerio de Sanidad y Consumo en su Plan Nacional de Acciones Preventivas de los efectos de los excesos de temperaturas sobre la salud ${ }^{35}, \mathrm{y}$ 
tomamos como referencia el percentil 95 de las serie completa (1975-2003) de temperaturas máximas diarias de la estación Toledo "Compuesta" $\left(36,5^{\circ} \mathrm{C}\right)$, el número de días que lo superan ha pasado de 84 en la década 1975-1984 a nada menos que 241 en la siguiente y 184 días en la tercera (ésta con un año menos). Por tanto, no se descarta que esto pudiera haber influido de alguna manera sobre la evolución obtenida.

No se dispone de datos sobre contaminación atmosférica que abarquen toda la geografía de CLM puesto que las estaciones medidoras se concentran en las zonas urbanas más importantes, sin que éstas puedan representar la exposición de la población regional o provincial, teniendo en cuenta su baja densidad y dispersión geográfica. Sin embargo, hay que señalar que no existen grandes núcleos urbanos o industriales en la Región por lo que la posible influencia de la contaminación atmosférica estaría muy limitada a determinadas ciudades, sin suficiente peso sobre la mortalidad regional o provincial como para alterar de forma significativa la asociación temperatura- mortalidad. No obstante, debe reconocerse este hecho como una limitación del estudio.

En todo caso, de los resultados obtenidos se extrae la información de que la relación de la temperatura ambiental con la mortalidad varía en el transcurso del tiempo de un modo que puede ser diferente según las ciudades o regiones implicadas, por lo que es necesario abordar estudios específicos antes que extrapolar conclusiones, ni siquiera entre países o regiones de similar desarrollo socio- económico. Por tanto, es preciso caracterizar dicha relación en unidades territoriales apropiadas, como provincias en el caso de CLM. Al mismo tiempo, esta dinámica temperatura- mortalidad nos indica que conviene revisar periódicamente los planes que se hayan establecido para prevenir los efectos del calor sobre la población, implantados como consecuencia de la ola de calor que afectó a Europa en 2003 27,36,37.
Cambios producidos en la población como su envejecimiento o movimientos migratorios significativos podrían dejar obsoletas las medidas preventivas adoptadas. No hay que olvidar que las proyecciones sobre población que se realizan para España insisten en la fuerte tendencia hacia el envejecimiento a medio plazo de la población ${ }^{38}$, como ha advertido recientemente el Departamento de Asuntos Económicos y Sociales de Naciones Unidas.

Si a una población envejecida le sumamos lo que debido al cambio climático los expertos pronostican como un aumento de la frecuencia e intensidad de las olas de calor, especialmente en el sur de Europa, las consecuencias podrían ser nefastas si no se actúa con previsión.

\section{BIBLIOGRAFÍA}

1. Mackenbach JP, Looman CW, Kunst AE. Air pollution, lagged effects of temperature and mortality: The Netherlands 1979-87. J Epidemiol Community Health 1993; 47(2):121-6.

2. Saez M, Sunyer J, Castellsague J, Murillo C, Anto JM. Relationship between weather temperature and mortality: a time series analysis approach in Barcelona. Int J Epidemiol 1995; 24(3):576-82.

3. Ballester F, Corella D, Pérez-Hoyos S, Saez M, Hervas A. Mortality as a function of temperatura. A study in Valencia, Spain, 1991-1993. Int J Epidemiol 1997; 26(3):551-61.

4. Kan HD, Jia J, Chen BH. Temperature and daily mortality in Shanghai: a time-series study. Biomed Environ Sci 2003; 16(2):133-9.

5. El-Zein A, Tewtel-Salem M, Nehme G. A timeseries analysis of mortality and air temperature in Greater Beirut. Sci Total Environ 2004; 330(13):71-80.

6. Douglas AS, Al-Sayer H, Rawles JM, Allan TM. Seasonality of disease in Kuwait. Lancet 1991; 337(8754):1393-7.

7. Curriero FC, Heiner KS, Samet JM, Zeger SL, Strug L, Patz JA. Temperature and mortality in 11 cities of the eastern United States. Am J Epidemiol 2002; 155(1):80-7. 
8. The Eurowinter Group. Cold Exposure and winter from ischaemic heart disease, cerebrovascular disease, respiratory disease, and all causes in warm and cold regions of Europe. Lancet 1997; 349: 1341-6.

9. Hajat S, Armstrong BG, Gouveia N, Wilkinson P. Mortality displacement of heat-related mortality: a comparison of Delhi, Sao Paulo and London. Epidemiology 2005; 16(5):613-20.

10. Sakamoto-Momiyama M: Seasonality of human mortality. Tokyo University Press 1977.

11. Davis RE, Knappenberger PC, Michaels PJ, Novicoff WM. Changing heat-related mortality in the United States. Environ Health Perspect 2003; 111(14):1712-8.

12. Michelozzi P, De SM, Accetta G, de'Donato F, Kirchmayer U, D'Ovidio M, Perucci CA. Temperature and summer mortality: geographical and temporal variations in four Italian cities. J Epidemiol Community Health 2006; 60(5):41723.

13. De Castro M, Martín-Vide J, Alonso S. El Clima de España: Pasado, Presente y Escenarios de Clima para el Siglo XXI. In: Moreno JM (eds). Evaluación Preliminar General de los Impactos en España por Efecto del Cambio Climático. Misterio de Medio Ambiente, Madrid; Universidad de Castilla-La Mancha 2005. Disponible en: "http://www.mma.es/oecc" y "http://ecce.uclm.es"

14. Kalkstein LS, Greene JS. An evaluation of climate/mortality relationships in large U.S. cities and the possible impacts of a climate change. Environ Health Perspec 1997; 105(1):84-93.

15. Gaffen DJ, Ross RJ. Increased summertime heat stress in the U.S. Nature 1998; 396:529-30.

16. Mirón Pérez IJ, Montero Rubio JC, CriadoAlvarez JJ, Gutierrez Ávila AG, Paredes Beato BD, Mayoral Arenas AS y Linares Gil C. Tratamiento y estudio de series de temperatura para su aplicación en salud pública. El caso de Casti1la-La Mancha. Rev Esp Salud Publica 2006; 80(2):113-24.

17. Box GE, Jenkins GM y Reinsel C. Time series analysis. Forecasting and control. Englewood. Prentice Hall 1994.

18. Miron IJ, Criado-Alvarez JJ, Diaz J, Linares C, Mayoral S, Montero JC. Time trends in minimum mortality temperatures in Castile- La Mancha (Central Spain): 1975-2003. Int J Biometeorol (En Prensa) 2007.
19. Davis RE, Knappenberger PC, Novicoff WM, Michaels PJ. Decadal changes in summer mortality in U.S. cities. Int J Biometeorol 2003; 47(3):166-75.

20. Carson C, Hajat S, Armstrong B, Wilkinson P. Declining vulnerability to temperature-related mortality in London over the 20th century. Am J Epidemiol 2006; 164(1):77-84.

21. Keatinge WR, Donaldson GC, Cordioli E, Martinelli M, Kunst AE, Mackenbach JP, Nayha S, Vouri I. Heat related mortality in warm and cold regions of Europe: observational study. BMJ 2000; 321(7262):670-3.

22. O'Neill MS, Zanobetti A, Schwartz J. Modifiers of the temperature and mortality association in seven US cities. Am J Epidemiol 2003; 157(12):107482.

23. El-Zein A, Tewtel-Salem M. On the association between high temperature and mortality in warm climates. Sci Total Environ 2005; 343(1-3):273-5.

24. Sakamoto-Momiyama M. Changes in the seasonality of human mortality: a medico-geographical study. 1978; Soc Sci Med 12(1D):29-42.

25. Basu R, Samet JM. Relation between Elevated Ambient Temperature and Mortality: A Review of the Epidemiologic Evidence. Epidemiol Rev 2002; 24(2):190-202.

26. Diaz J, Garcia R, Velazquez de CF, Hernandez E, Lopez C, Otero A. Effects of extremely hot days on people older than 65 years in Seville (Spain) from 1986 to 1997. Int J Biometeorol 2002; 46(3):145-9.

27. Conti S, Meli P, Minelli G, Solimini R, Toccaceli V, Vichi M, Beltrano C, Perini L. Epidemiologic study of mortality during the Summer 2003 heat wave in Italy. Environ Res 2005; 98(3):390-9.

28. Montero Rubio JC, Mirón Pérez IJ, Díaz Jiménez J, Alberdi Odriozola JC. Influencia de variables atmosféricas sobre la mortalidad por enfermedades respiratorias y cardiovasculares en los mayores de 65 años de la Comunidad de Madrid. Gac Sanit 1997; 11(4):164-70.

29. Diaz J, Jordan A, Garcia R, Lopez C, Alberdi JC, Hernandez E, Otero A. Heat waves in Madrid 1986-1997: effects on the health of the elderly. Int Arch Occup Environ Health 2002; 75(3):163-70.

30. Alberdi JC, Diaz J, Montero JC, Mirón I. Daily mortality in Madrid community 1986-1992: relationship with meteorological variables. Eur J Epidemiol 1998; 14(6):571-8. 
31. Diaz J, Garcia R, Linares C, Tobias A, Prieto L. Mortality impact of extreme winter temperatures. Int J Biometeorol 2005; 49(3):179-83.

32. Kunst AE, Looman CW, Mackenbach JP Outdoor air temperature and mortality in The Netherlands: a time-series analysis. Am J Epidemiol 1993; 137(3):331-41.

33. Braga AL, Zanobetti A, Schwartz J. The time course of weather-related deaths. Epidemiology 2001; 12(6):662-7.

34. Pattenden S, Nikiforov B, Armstrong BC. Mortality and temperature in Sofia and London. J Epidemiol Community Health 2003; 57(8):62833.

35. Plan Nacional de Acciones Preventivas de los efectos del exceso de temperaturas sobre la salud. Ministerio de Sanidad y Consumo, Madrid 2007. Disponible en: "http://www.msc.es/ciudadanos/saludAmbLaboral/planAltasTemp/2007/docs/planDefinitivoOlaDeCalor2007.pdf"

36. Diaz J, Garcia-Herrera R, Trigo RM, Linares C, Valente MA, De Miguel JM, Hernandez E. The impact of the summer 2003 heat wave in Iberia: how should we measure it? Int J Biometeorol 2006; 50(3):159-66.

37. Le Tertre TA, Lefranc A, Eilstein D, Declercq C, Medina S, Blanchard M, et al. Impact of the 2003 heatwave on all-cause mortality in 9 French cities. Epidemiology 2006; 17(1):75-9.

38. World Population Prospects: The 2006 Revision. United Nations, New York 2005. Disponible en: "http://www.un.org/esa/population/publications/wp p2006/wpp2006.htm" 